\title{
Philosophiques
}

\section{Être quelque chose}

\section{Charles Travis}

Volume 45, numéro 1, printemps 2018

URI : https://id.erudit.org/iderudit/1048623ar

DOI : https://doi.org/10.7202/1048623ar

Aller au sommaire du numéro

Éditeur(s)

Société de philosophie du Québec

ISSN

0316-2923 (imprimé)

1492-1391 (numérique)

Découvrir la revue

Citer ce document

Travis, C. (2018). Être quelque chose. Philosophiques, 45(1), 223-238.

https://doi.org/10.7202/1048623ar

Ce document est protégé par la loi sur le droit d'auteur. L'utilisation des services d'Érudit (y compris la reproduction) est assujettie à sa politique d'utilisation que vous pouvez consulter en ligne.

https://apropos.erudit.org/fr/usagers/politique-dutilisation/
Cet article est diffusé et préservé par Érudit.

Érudit est un consortium interuniversitaire sans but lucratif composé de l’Université de Montréal, l'Université Laval et l'Université du Québec à Montréal. Il a pour mission la promotion et la valorisation de la recherche. https://www.erudit.org/fr/ 


\title{
Être quelque chose
}

\author{
CHARLES TRAVIS \\ King's College London
}

Au tournant du millénaire, Frege, d'un ennemi, est devenu un ami. Tournant le dos à la tradition orale, je le lisais véritablement pour la première fois. Une bonne part de la philosophie - et certainement pas seulement ou principalement de la philosophie du langage - est alors rapidement devenue beaucoup plus claire. C'est ainsi que, dans ce texte, je me concentrerai sur deux problèmes soulevés par Jocelyn Benoist dans L'adresse du réel.

La clarté qu'offre Frege à la philosophie en général tient pour une bonne part à l'aspect suivant: le thème central de la philosophie de Frege est le fait d'être vrai comme tel, c'est-à-dire ce qui s'ensuit de cette seule notion pour ce que c'est que penser les choses comme étant ce qu'elles sont («les lois de la vérité ", comme il désigne lui-même cette question). Ce qui rend d'abord possible une telle notion, c'est le phénomène consistant à représenter quelque chose comme étant quelque chose - c'est-à-dire le fait de représenter-comme [representing-as]. Cette possibilité est ouverte par la distinction qui existe entre ce premier sens du verbe représenter et la notion de représentation factive [factive representing]. Ces rides sur le front de Pia peuvent représenter plusieurs années de vie partagées avec Sid. Mais ce n'est le cas que si elle a bien partagé plusieurs années de vie avec Sid. Si tel n'est pas le cas, quoi que représentent ces lignes sur le front, ce ne sera en tout cas pas cela. Tandis qu'il n'y a aucun problème à représenter Pia comme ayant partagé plusieurs années de vie avec Sid même si ce n'est pas le cas. La fausseté est ainsi rendue possible et, par conséquent, la vérité également.

Frege s'intéresse à la forme que prend ce phénomène seulement en tant que c'est ce qui s'ensuit du fait d'être vrai. Il fait donc abstraction, pour cerner l'objet de son étude - le représenter-comme - de tout représentant-comme historique - de tous les penseurs ou de toutes les instances historiques qui s'engagent ainsi avec le monde. Par ce processus d'abstraction, on perd certains aspects du phénomène. Mais, précisément, en perdant quelque chose, le phénomène gagne aussi en clarté. En réinsérant le phénomène abstrait dans le monde, dès lors armés d'une idée claire de ce que nous réinsérons, nous sommes conduits à reformuler de manière particulièrement bénéfique plusieurs questions qui taraudent les philosophes, qu'elles soient métaphysiques ou qu'elles concernent la pensée ou la condition humaine.

Qu'on nous permette ici une remarque terminologique. Le mot "pensée» [thought] en anglais dérive de deux nominalisations possibles du verbe «penser». Il peut désigner un certain phénomène général de pensée, une activité des penseurs, un objet d'étude de la psychologie. Ou encore, il peut

PHILOSOPHIQUES 45/1 — Printemps 2018, p. 223-238 
désigner l'objet de l'activité de pensée, c'est-à-dire un état possible des choses, une façon de les concevoir. Chacune de ces nominalisations, à tout le moins initialement, est un nom massif ${ }^{1}$ qui attend d'être découpé en unités dénombrables [countables]. Afin d'éviter toute confusion, je distinguerai entre l'une et l'autre en employant pensée $\psi$ et pensée $\lambda$. Nous nous préoccuperons presque exclusivement de la pensée $\lambda$ dans ce qui suit.

I. Le premier problème dont je discuterai survient en second au cours de l'ouvrage, mais il est prioritaire pour Frege comme pour moi. Il s'exprime ainsi:

Quelque chose de Venus-même - un aspect de sa réalité — est thématisé dans la pensée (la vérité) qu'elle est l'étoile du soir. Est-ce pour autant une raison pour confondre le sens et l'être de la chose? ... Je ne crois pas que les 'sens' en un sens frégéen adapté ... puissent être tenus pour des propriétés des choses elles-mêmes. Je pense qu'il y a une différence de principe entre la grammaire de 'détermination,' notion que j'applique au niveau du sens, et celle de la propriété. (97)

Pour anticiper un peu, c'est précisément cette confusion du sens avec la chose que cherche à éviter Frege en distinguant entre Merkmale et Eigenschaften. Mais, tout d'abord, comment commettre cet appariement fautif? La forme qu'il prend, que ce soit ici ou ailleurs, doit encore être élucidée.

Frege choisit ce qu'il appelle une pensée (Gedanke) en tant que protagoniste central de l'activité de pure représentation: c'est une unité dénombrable dans laquelle s'articule la pensée $\lambda$. Les autres protagonistes intervenant dans la pensée $\lambda$ devront être identifiés en fonction de leur relation à cette entité dénombrable. Une pensée, nous dit Frege, est précisément ce grâce à quoi la question de la vérité ne peut jamais se poser. Elle fait dépendre la vérité, de manière déterminée, de l'état des choses. On parle de deux pensées différentes chaque fois qu'il y a deux manières différentes pour l'état des choses d'importer à l'égard de la vérité, c'est-à-dire deux demandes différentes à son endroit. C'est une idée qui, en soi, est lourde de sens, et que nous n'explorerons pas davantage ici.

Une pensée se distingue par le fait qu'elle est absolument vraie (ou fausse). "Vrai absolument» se distingue ici de «vrai de». Ce qui représente une chose comme étant impétueuse peut la représenter véridiquement si cette chose est Maurice, ou faussement s'il s'agit de Moore. Il pourrait être

1. Note de la traductrice: L'auteur fait référence à une distinction en linguistique entre nom massif (ou nom non-comptable) [mass noun] et nom comptable [count noun]. Le nom comptable désigne un nom qui peut être quantifié et peut être employé au pluriel et au singulier (i.e. un chat, deux chats, trois chats). Le nom massif désigne un nom qui dénote une chose ne pouvant pas être comptée, ou un nom qui dénote une chose qui n'est habituellement pas comptée mais qui peut l'être dans certains emplois. Un même nom peut être comptable dans certains emplois et non-comptable dans d'autres (i.e. en anglais le nom massif [paper] qui devient [printing papers, blotting papers, wrapping papers]). 
vrai d'affirmer de Benno qu'il est impétueux, mais faux de l'affirmer de Moore. Ce qui représente une pensée ne contient pas ce type de dépendance à autre chose. Si la pensée est que Moore est impétueux, sa vérité ou sa fausseté ne dépend pas de qui, ou de ce qui, opère la représentation. Toute pensée, qu'elle soit vraie ou fausse, fait dépendre la vérité de la même chose. Toute manière dont une pensée entière représente les choses (et dont dépend la vérité) est, respectivement, une manière pour la même chose d'être. La pensée selon laquelle Maurice est impétueux et le fait que le Discours de la méthode de Descartes a été publié l'année de la crise de la tulipe sont, à cet égard, identiques.

Mais de quoi la vérité peut-elle donc dépendre? Une première réponse possible serait "des choses", ou encore, plus exactement, "de l'état des choses" [how/the way things are]. Comme cela arrive parfois en anglais, cette formulation adopte un pluriel trompeur [schein]. "Les choses vont mal'». Quelles choses? (De manière similaire, «les faits» sera souvent schein-pluriel.) Tout comme le terme pensée $\lambda$ plus tôt, «les choses » doit être lu comme un nom massif. Il en va de même, corrélativement, de notre usage de l'expression 'l'état des choses'. Quel est cet état des choses? Inutile d'attendre ici une réponse. L'ensemble générique sera articulé en manières dénombrables qu'ont les choses d'être. Pour signifier le caractère générique du terme 'choses', j'emploierai 'les choses m $^{\text {' }}$.

Grâce à une pensée, nous articulons l'ensemble générique, la pensée $\lambda$, en entités dénombrables qui seront dénombrées selon le principe de Frege sus-mentionné. L'ensemble s'articule, corrélativement, en manières de penser l'état des choses (en manières possibles dont les choses $s_{\mathrm{m}}$ peuvent être). L'état des choses, ou la manière dont les choses sont, s'articule donc en un domaine propre, issu du domaine constitué par l'ensemble des états possibles qu'il peut prendre (les états qui sont ceux dans lesquels se trouvent les choses).

Précisons maintenant que nous appellerons respectivement les deux pôles du représenter-comme (direction sujet à objet) le représenté [the depicted] (e.g. Zoe, ou les choses ${ }_{\mathrm{m}}$ ) et la représentation [the depiction] (e.g. comme étant impétueuse, ou comme étant telle qu'est Zoe). Les pensées représentent, mais pas au sens où le ferait un penseur. Une pensée identifie une manière distincte dont un penseur peut représenter les choses. L'exprimer, c'est représenter les choses ainsi. On peut alors considérer qu'il s'agit de la représentation du représenté (les choses $\mathrm{s}_{\mathrm{m}}$ ) par cette représentation.

Suivant ce portrait, la vérité semble être l'affaire exclusive de deux éléments. Le premier élément prenant part à cette entreprise, c'est la représentation (ou le fait qu'elle représente). Son rôle est d'exiger ou de demander [demand] quelque chose du second élément $\left(\right.$ les choses $_{\mathrm{m}}$ ). Le rôle du second élément, en étant ce qu'il est, est de satisfaire, ou non, à cette exigence. L'exigence en question est d'être tel qu'il est représenté, et la conformité à cette exigence s'appelle la vérité. 
Il y a toutefois une condition à la conformité du premier élément intervenant dans cette affaire. Elle tient dans l'idée de conformité possible, et cette disposition est précisément ce qui distingue le représenter-comme de la représentation factive. Cette condition apparaît précisément dans le passage de la pensée $\lambda$ aux pensées, et, corrélativement, dans le passage de la manière dont les choses sont (ou ne sont pas) aux diverses manières qu'elles ont d'être ou de ne pas être. La manière dont les choses sont s'articule inter alia en un état de choses précis selon lequel la crise des tulipes a éclaté en I637, mais avec un résidu substantiel. Ce n'est pas que les choses sont telles qu'elles sont seulement si la crise a éclaté cette année-là. Pour une large part, le fait que la crise a effectivement éclaté en I 637 laisse ce résidu indéterminé. Autrement dit, ce résidu pourrait varier (ou aurait pu varier) alors même que le fait que la crise a éclaté à ce moment demeurait avéré. Ce que cet état possible des choses - l'éventualité de la crise de I637 - doit fixer, c'est donc la portée que la variation dans l'état des choses ${ }_{\mathrm{m}}$ doit avoir pour permettre que cet état demeure le même, ou pour éliminer cette articulation entre les deux. Autrement dit, voici ce que nous obtenons à partir de cette illustration: L'état possible dans lequel les choses ${ }_{\mathrm{m}}$ peuvent être possède, intrinsèquement, une généralité propre [proprietary generality]: elle est une généralisation de cas particuliers (et, dans le cas d'une pensée complète, une généralisation de cas possibles) de telle sorte qu'elle permet de distinguer les moments où l'état des choses serait une instance pertinente de la généralité en question, et ceux où cet état n'en est pas une. C'est en raison de cette généralité qu'une pensée, ou la manière dont elle représente les choses ${ }_{\mathrm{m}}$, peut adresser une exigence à l'égard de l'état des choses ${ }_{\mathrm{m}}$, qui puisse lui peut être adressée qu'elle soit (ou non) satisfaite. C'est précisément ainsi que la question de la vérité fait son entrée sur scène.

Une pensée, nous dit Frege (Kernsatz 4, date inconnue), contient toujours une généralité sous laquelle elle fait tomber les choses ${ }_{\mathrm{m}}$. (Frege ajouterait que la pensée est identifiée comme la pensée qu'elle est par cette généralité.) Nous voyons maintenant que cette généralité marque la manière dont cette pensée représente comment sont les choses ${ }_{\mathrm{m}}$. Si les choses ${ }_{\mathrm{m}}$ se rangent sous cette généralité, alors l'état des choses ${ }_{\mathrm{m}}$ est une des choses qui y tombent, c'est-à-dire une manière pour les choses d'être de la manière dont elles sont décrites, parmi une infinité d'autres manières possibles de l'être. Mutatis mutandis si les choses ne sont pas de cette manière. Si le ciel est bleu, il le sera que la cuisine soit chaude ou froide.

L'exigence qui est adressée à la représentation (rôle du premier élément) en est une qui n'est pas adressée au représenté (rôle du second élément). L'exigence de conformité n'est pas la même pour les deux rôles. Frege spécifie cette différence au moyen de la distinction entre Merkmal et Eigenschaft (propriété). Le Merkmal d'une généralité est une exigence (déterminable) qu'elle impose à son instanciation. Si la généralité en question est le fait pour une chose d'être un homme, un Merkmal pourrait être le fait d'être 
incapable de voler et bipède. Si une chose instanciait cette généralité, alors elle serait incapable de voler et bipède. C'est la chose qui serait ainsi, et non la généralité elle-même. Il en va de même pour la généralité $d u$ fait pour Sid d'être un homme. Ce qui en serait une instanciation serait du même coup une instanciation de la généralité du fait pour Sid d'être incapable de voler et $d u$ fait pour Sid sd'être bipède. C'est l'instanciation de cette première généralité, ou encore la manière dont sont les choses $_{\mathrm{m}}$, qui serait une instanciation des deux suivantes, et non pas la généralité du fait pour Sid d'être un homme. Telle est la structure du représenter-comme.

Ainsi, donc, de la différence grammaticale sur laquelle Benoist insiste avec raison. Que pourrait-il être en train de nier ce faisant? Pour répondre à cette question, il sera utile de passer maintenant de l'élément qui joue le rôle principal dans le fait d"être vrai [being true] chez Frege aux autres éléments qui se définissent par rapport à lui. Nous aurons besoin des concepts suivants: celui d'une véritable partie de pensée (une partie qui n'est pas ellemême une pensée, mais une sous-pensée [subthought]); celui d'une autre partie prédicative, celui de concept, et enfin celui d'objet.

Commençons d'abord avec le concept de sous-pensée. Une sous-pensée remplit partiellement la mission définitoire d'une pensée. Une pensée fait dépendre la vérité de manière déterminée de la manière dont les choses sont. Une sous-pensée, quant à elle, en fait dépendre la vérité de façon partielle. La décomposition (d'une pensée) consiste donc seulement en un ensemble de réalisations partielles de ce que la pensée elle-même fait, et dont le travail conjoint est, en soi, exactement ce que la pensée entière réalise. Chaque élément obtenu au terme de la décomposition, s'il n'est pas luimême une pensée, est une sous-pensée. Une remarque ici: ce que fait une sous-pensée ne peut être fait que dans le cadre d'une décomposition. Si, de la pensée selon laquelle Sid a terminé sa poutine, nous isolons une partie qui fait dépendre la vérité de ce qu'est Sid (ou de ce qu'il a fait), il n'est toutefois pas possible de rencontrer un élément qui ne fasse que cela, de lui-même fertig. Une seconde remarque : étant donné ce en quoi consiste la décomposition, la même pensée peut être décomposée de plusieurs manières différentes. C’est même le plus souvent le cas, selon Frege.

Toute pensée peut être décomposée en sous-pensées. En voici une explication. Lorsque la manière dont les choses $\mathrm{m}_{\mathrm{m}}$ sont (ou l'état des choses $\mathrm{m}_{\mathrm{m}}$ ) s'articule en manières particulières qu'ont les choses ${ }_{\mathrm{m}}$ d'être, il y a ipso facto, un résidu. Ce résidu nous livre bien plus d'informations que ne nous en livre ce qui est articulé. Cette perte d'informations est compensée par le gain suivant: l'information qui nous reste est utilisable sous une nouvelle forme. L'information dont nous disposons alors peut avoir une incidence, dont nous pouvons juger, sur l'état des choses qui se trouve dans des régions autrement hors de notre portée. Si Sid en est à son troisième apéritif, il ne faut pas trop s'en approcher si l'on veut s'épargner une harangue. Ce rapport repose sur un principe de similitude. En élaborant, à partir de 
l'état des choses, la manière dont une pensée entière représente les choses ${ }_{\mathrm{m}}$, nous déterminons un certain sens de la similitude en fonction duquel une chose demeurera toujours la même bien qu'elle subisse une certaine gamme de changements. Pareillement, en élaborant une sous-pensée à partir d'une pensée, nous déterminons un certain sens de la similitude en fonction duquel on pourra (ou non) trouver quelque chose de commun aux manières dont deux pensées différentes représentent les choses ${ }_{\mathrm{m}}$. Ainsi, nous pouvons parvenir à reconnaître des façons d'être pour les choses (à certains égards) qui nous auraient autrement échappé. C’est donc grâce aux généralités que l'état des choses ${ }_{\mathrm{m}}$ en vient à importer pour décider ce que nous devons faire.

Frege observe que chaque décomposition doit contenir au moins un élément qui n'est pas lui-même une pensée, mais qui joue le même rôle, c'està-dire celui de représenter-comme. (Attention: il s'agit là d'une version aseptisée du propos de Frege.) Ce rôle consiste à représenter-comme: la pensée que Benno radote représente les choses ${ }_{\mathrm{m}}$ d'une certaine manière. Cette pensée contient une part identifiable qui fait dépendre la (sa) vérité de l'identité de celui qui radote. La partie en question représente Benno comme un radoteur. Chaque décomposition doit procéder ainsi: si la tâche du représenter-comme n'était pas déléguée par la pensée entière à quelque souspensée lors de la décomposition, le travail conjoint des éléments de ladite décomposition ne pourrait précisément pas faire ce que la pensée entière réalise. Une véritable sous-pensée prédicative doit elle-même être engagée dans la représention-comme. Tel est le second élément de notre liste, identifié en fonction de sa relation avec la pensée entière.

L'élément suivant sur notre liste est celui de concept (au sens que nous lui donnons maintenant); il se situe à un pas de la sous-pensée prédicative, et se trouve plus éloigné de la notion de pensée. Si, comme en logique, nous nous intéressons à ce qu'il en est de la pensée en vertu de ce qu'est le seul fait d'être vrai, une bonne partie de la pensée selon laquelle Benno est moralisateur ne nous intéressera pas. Rien dans le fait qu'il soit moralisateur ne présentera aucun intérêt. Du point de vue de la logique, la seule chose qui importe est qu'un certain 'quelque chose' a été prédiqué d'un autre 'quelque chose'. $\mathrm{Si}$, dans la sous-pensée prédicative, nous faisons abstraction de tout ce qui n'importe pas de cette manière, il ne reste que l'occurrence d'une certaine prédication - la forme à nu, pour ainsi dire, de cette prédication. Les détails qu'elle omet - et que nous avons abstraits de la généralité 'le fait d'être moralisateur' - identifient un concept: le concept d'une chose qui est ainsi. Réciproquement, le concept se détache de l'engagement que prend la sous-pensée en représentant-comme, c'est-à-dire de la forme de la prédication. Un concept ne représente rien comme étant quelque chose. Mais ce type d'engagement (le simple fait de représenter-comme) peut s'attacher à sa généralité, ou à celle qu'il forme. Cette caractéristique permet simplement au contenu de ce type de représentation (le représenter-comme), autrement dit à une manière dont une chose peut être, de représenter ainsi quelque 
chose. Un concept, pourrions-nous dire, est donc le contenu possible d'une sous-pensée prédicative.

Qu'en est-il, enfin, de l'objet? Le terme évoque intuitivement des choses avec lesquelles nous pouvons entrer en collision [collidables], ou qui peuvent bloquer notre chemin, comme par exemple Sid, ou encore un soulier sur la dernière marche de l'escalier. Or, du point de vue de la logique, un objet désigne tout ce qui peut être combiné avec un état possible d'une chose (dénombrable) pour former une manière dont une pensée peut représenter les choses - c'est-à-dire quelque chose dont la vérité peut directement dépendre. L'idée est la suivante: tout ce qui peut se combiner avec l'un de ces états possibles pourra l'être avec un nombre indéfini d'autres. (Là encore, si nous procédons à une décomposition, nous identifierons différentes manières pour deux pensées d'être identiques.) Un objet est donc, de manière intrinsèque, ce qui peut se répéter. Lorsqu'il y a un objet, il est toujours possible de le rencontrer à nouveau. Pour le dire autrement, il n'y a d'objet que lorsqu'il y a le concept d'être (identique à) celui-ci. En somme, l'identité constitue la relation fondamentale que tous les objets (et seulement eux) entretiennent (à l'égard d'eux-mêmes, et d'eux seuls, bien sûr).

Nous arrivons maintenant à une zone d'ombre dans la pensée de Frege. Frege emploie deux notions de manière plus ou moins interchangeable, comme si ce qui correspondait à la première correspondait également, eo ipso, à la seconde. L'une des notions est Erganzungsbedurftigkeit ou Ungesattigheit (élément non saturé). La seconde est celle de nature prédicative. Dans notre liste d'items, la sous-pensée correspond le plus clairement à ce besoin d'être saturé (ou complété): elle ne peut réaliser ce qu'elle fait que dans le contexte d'une décomposition; il faut donc qu'une certaine saturation ait lieu pour former un certain tout, en l'occurrence ici, une pensée. Ce dont la logique a besoin, pour sûr, c'est de véritables sous-pensées types (par exemple: la prédication du caractère moralisateur de quelqu'un). Ces souspensées sont complétées de différentes manières dans différentes pensées, mais l'exigence de complétude demeure la même. Cette nature prédicative est ce que possèdent les sous-pensées prédicatives en faisant ce qu'elles font, à savoir prédiquer quelque chose de quelque chose.

Nous pouvons maintenant reformuler un point rencontré plus tôt: toute décomposition doit contenir une sous-pensée prédicative. Pourquoi? Parce qu'une part du produit de la décomposition doit remplir la même tâche que la pensée entière, à savoir celle de représenter-comme. Une souspensée ne remplit cette tâche, ou ne fait la même chose (selon le sens requis de même), que si elle prédique quelque chose. Par conséquent, il en va de même pour la pensée qui accomplit cette tâche. La partie issue de la décomposition, bien sûr, prédique quelque chose d'un objet, tandis que la pensée entière prédique quelque chose de l'ensemble générique des choses ${ }_{\mathrm{m}}$ - avant qu'il ne soit découpé en objets. Jusqu'ici, nous avons considéré que le concept et, par conséquent aussi, la sous-pensée prédicative était une 
manière dont peut être une chose. Si la nature prédicative en constitue la pierre de touche, on peut généraliser la notion: nous pouvons parler d'un concept à n-place (ou d'une manière d'être pour un nombre $n$ d'objets), mutatis mutandis pour les sous-pensées prédicatives. En fait, Frege a même besoin de cette idée s'il veut réellement être débarrassé de la distinction sujet-prédicat. Qu'on formule donc l'argument en ces termes: lorsqu'il est question de nature prédicative, nous pouvons parler en ces termes pour $\mathrm{n} \geq 0$.

Une pensée entière ne fait précisément pas ce qui ne peut être fait que dans le contexte d'une décomposition. C'est le point central de l'idée que la vérité dépend de l'état des choses. Mais elle possède une nature prédicative si, comme décrit plus haut, c'est ce à quoi correspond le fait de faire une activité similaire. Auquel cas l'exigence de saturation (ou de complétude) et la nature prédicative sont disjointes.

Au niveau syntaxique, nous distinguons entre le groupe nominal et le sujet (de la phrase). Le groupe nominal est une notion catégorielle. Il s'agit d'un type de locution, identifié comme tel par sa structure interne, indépendamment du lieu de son occurrence dans une phrase. Le sujet, à l'inverse, (cette notion que Frege souhaite éliminer de la logique) est intrinsèquement relationnel: un sujet est un groupe nominal qui se rapporte d'une manière particulière au reste de la phrase dans laquelle il apparaît. Il est donc intrinsèquement sujet de cette phrase. Comment la distinction entre le relationnel et le catégoriel s'applique-t-elle aux éléments que nous avons introduits pour rendre compte de cette chose dénombrable qu'est la pensée ? Pour commencer, une sous-pensée prédicative est, à première vue, une notion relationelle: la sous-pensée prédicative réalise une opération partielle, dans le cadre d'une décomposition, par rapport à l'opération entière d'une pensée (donnée); elle est donc reliée d'une certaine manière à la décomposition entière. Certaines complications peuvent apparaître lorsqu'une pensée entière, ou un contenu de pensée, est incorporé à un autre, mais nous suspendrons ces considérations ici. Dans tous les cas, il est impossible qu'une sous-pensée prédicative apparaissant au terme d'une décomposition ait alternativement le rôle de prédiquer (ou de représenter-comme) et, éventuellement, un autre rôle.

Qu'en est-il maintenant du concept (à une place) [1-place concept]? Nous avons introduit ce type de concept comme une sorte d'abstraction réalisée à partir de la sous-pensée prédicative. Il y est précisément fait abstraction de la nature prédicative de cette dernière; il n'est pas impliqué dans la tâche de représenter-comme. Il reste néanmoins vrai que nous n'avons à proprement parler de concept que lorsque quelque chose peut jouer le rôle du contenu d'une sous-pensée prédicative. Mais cette idée semble revenir à définir une catégorie plutôt qu'une notion relationnelle - quelque chose, donc, qui s'apparente au groupe nominal dans la phrase, distribué d'une certaine manière dans un corpus d'articulations de pensées $\wedge$, mais qui ne se tient néanmoins pas toujours nécessairement dans la même relation avec tous les ensembles avec lesquels il entre en relation. 
Il est exact qu'aucune chose avec laquelle nous pouvons entrer en collision [collidables], ni aucune chose spatio-temporelle, ne peut jouer le rôle d'un concept dans une sous-pensée prédicative. Pas plus ne trouverons-nous aucun concept dans l'espace-temps. Nous avons donc bien ici deux notions catégorielles exclusives: aucune entité avec laquelle nous pouvons entrer en collision (ou aucune chose mondaine) n'est un concept et aucun concept n'est une chose avec laquelle nous pouvons entrer en collision (ou une chose mondaine). Or, comme nous l'avons vu, la catégorie objet (s'il s'agit d'une catégorie) est beaucoup plus large. Comme c'est le cas avec un concept, un objet désigne une chose qui peut occuper un rôle particulier dans une pensée (ou dans des pensées): un objet doit pouvoir former, avec un concept à une place (ou, plus exactement, avec ce dont il est le concept - une certaine manière pour les choses d'être), une manière dont une pensée entière peut représenter l'être des choses. Nous pourrions donc concevoir l'objet en fonction du rôle que l'objet doit être capable de jouer. Or, jusqu'ici, rien n'empêche un item distinct des choses avec lesquelles nous pouvons entrer en collision, comme nous avons dit que l'était le concept, de jouer ce rôle; ni donc non plus n'empêche ce qui joue ce rôle d'être un concept.

Un concept est, ou est identifié par, une certaine généralité: celle d'être ce dont il est le concept. Lorsqu'il est uni à un caractère prédicatif pour former une sous-pensée prédicative, cette généralité est sollicitée : elle devient la généralité de la sous-pensée. Or ceci ne revient pas exactement à affirmer qu'il n'est question d'un concept que lorsque cette généralité est ainsi sollicitée. Le concept ne possède pas de nature prédicative. $I l$ n'est pas engagé dans la tâche de représenter-comme - précisément pas. Le concept luimême, ou ce dont il est le concept, est, pour ainsi dire, 'erganzungsfähig': susceptible d'être complété d'une part (par une prédication) pour former une sous-pensée prédicative, d'autre part (par un objet) pour former un état possible des choses et donc, pour un objet donné et par rapport à lui, une manière dont les choses sont ou ne sont pas. Cette idée a peut-être impressionné Frege, mais Ergansungsfähigkeit n'est pas Erganzungsbedurftigkeit. Un concept n'est pas astreint à une exigence de complétude comme l'est la sous-pensée prédicative, ni cette exigence n'apparait-elle autrement qu'en vue du représenter-comme. Jusqu'ici, rien n'empêche qu'un concept occupe le rôle d'un objet dans la pensée, de la même manière que rien n'empêche un groupe nominal, en tant qu'il se distingue du groupe verbal, d'occuper la fonction d'objet direct.

Lorsque nous observons attentivement le phénomène du représentercomme, la première chose qui peut nous frapper est la différence fondamentale entre la tâche des deux partis de la relation. Être représenté comme ceci ou comme cela est somme toute très différent du fait d'être la représentation en question. Dans le représenter-comme, note Frege, une chose est présentée comme tombant sous une certaine généralité. Ce qui est instancié et ce qui instancie apparaissent ici comme deux choses de nature très différente. Si on 
observe d'abord le cas des pensées portant sur des choses avec lesquelles nous pouvons entrer en collision, cette différence de nature semblera simplement une différence entre ce qui possède un degré de généralité (ce qui peut être généralisé d'une manière particulière) et ce qui n'en possède pas. Mais si on dépasse le cas particulier des choses avec lesquelles nous pouvons entrer en collision et qu'on pense à l'objectité en général, la différence s'affiche plutôt en termes relationnels. Ce qu'il faut comprendre du rôle d'un objet (dans une pensée donnée), c'est que toute chose qui occupera ce rôle ne sera pas employée dans cette pensée pour former une partie de pensée comme l'est le concept pour former une sous-pensée prédicative.

Une manière possible d'être pour une chose - c'est-à-dire un concept - est ce qu'il nous reste de la sous-pensée prédicative lorsqu'on lui retire sa nature prédicative (le fait de prédiquer, de représenter-comme). De ce fait, le rôle du concept dans la sous-pensée prédicative est d'y apparaître avec une nature prédicative attachée. Supposons qu'une pensée se décompose, inter alia, en une sous-pensée responsable de faire dépendre la vérité de la manière dont tel ou tel objet $x$ est. Cette sous-pensée attache quelque chose à un concept, comme le ferait une sous-pensée prédicative. Or, cette chose qu'elle y attache ne sert pas à prédiquer, mais plutôt (bien sûr) à désigner. C'est ce qu'on pourrait appeler la 'nature désignative'. Face à un objet donné, la sous-pensée désignative l'attache à un concept qui est celui d'être cet objet précis. Supposons maintenant que cet objet soit un concept. Dans ce cas, le concept auquel la sous-pensée désignative le harnache serait celui d'être ce concept précis. Assurément, le concept désigné posséderait ou incarnerait une certaine généralité. Ce qui serait attendu de lui dans ce rôle, toutefois, n'est rien de plus que ce qui serait attendu d'une personne, d'un flétan ou d'un département de philosophie. Pour jouer son rôle dans cette pensée, comme ces autres choses, il n'a qu'à être précisément tel qu'il est. Sa généralité, son caractère généralisant, est ici inerte.

Telle est la différence entre la grammaire des choses et la grammaire de leur représentation (ou, selon les termes de Benoist, de leur détermination). Ici, deux choses sont liées. Premièrement, les limites de la généralité ne sont pas celles du non-saturé (ou de l'erganzungsbedurftig). Il y a des concepts à zéro place [zero-place concepts]. Il y a des pensées. Deuxièmement, ce qui est essentiel à l'objectité - ou, en termes relationnels, au rôle de l'objet n'est pas l'exigence d'être dépourvu de généralité, d'être une chose qui ne peut pas être instanciée, mais plutôt le fait de ne pas avoir de généralité sur laquelle s'appuie la pensée, ou une sous-pensée, pour former sa manière exclusive de représenter-comme. Ces points ont été négligés. Comment, une fois observés, peuvent-ils nous induire en erreur?

Qu'on prenne, par exemple, la pensée selon laquelle Benno est moralisateur. Supposons qu'il soit moralisateur, qu'il en soit le parangon, comme on en voit rarement. Nous dirons donc que la pensée est vraie. L'idée est la suivante: il doit y avoir quelque chose dans les choses $s_{\mathrm{m}}$, "dans le réel », qui 
la rende vraie. L'idée est raisonnable considérant ce que nous avons avancé jusqu'à présent sur les deux éléments impliqués dans la vérité. Et, en effet, cela s'avère exact: s'il est vrai que Benno est moralisateur, alors l'état des choses $_{\mathrm{m}}$ est l'une des choses qui peuvent valoir comme le fait d'être ainsi, et donc l'une des manières dont cela peut être vrai. Or, quelqu'un pourrait exiger plus. L'idée serait alors la suivante: il doit y avoir quelque chose dans la 'réalité' qui redouble - qui en soit comme le reflet, ou qui même partage — la structure de cette représentation, c'est-à-dire la pensée (et ce, même si ce qui est ainsi structuré est différent). Deux choses, toutes deux moralisatrices, le sont chacune à leur manière. Elles sont donc semblables selon un certain sens de 'semblable': semblables selon les limites admises par le concept 'être moralisateur'. Comme si la nature moralisatrice était une sorte de substance qui se retrouvait dans toutes les choses moralisatrices, formant avec elles un complexe structuré à la manière dont l'est la pensée selon laquelle telle chose est moralisatrice.

Ce type d'idée donne naissance à de mauvaises théories de la vérité. On la trouve dans les premiers textes de Russell, sous une certaine forme dans le Tractatus, et ailleurs assez souvent aussi, mais il s'agit d'une erreur - une erreur dans laquelle nous pouvons être entraînés, entre autres choses, par équivoque. En I 879, Frege suggère (I 879: 3-4) qu'on peut détacher le contenu d'une pensée de son engagement dans la tâche consistant à représenter-comme, de la même manière (comme nous l'avons noté) que peut l'être le contenu d'une sous-pensée prédicative. Cet engagement peut ensuite être rétabli, suggère Frege, au moyen d'un prédicat simple qui (de manière nécessaire) remplit seulement et précisément cette fonction sans ajouter de contenu. L'exemple de Frege était le suivant: 'La ruine d'Archimède sous la conquête de Syracuse.' Un autre exemple serait: 'La chute d'un moineau dans la soupe'. Ces locutions offrent deux lectures possibles. D'une part, elles rapportent un certain évènement historique, et donc elles ne rapportent rien sans la chute de ce moineau, ou la ruine d'Archimède. Une seconde lecture s'annonce (et mieux vaut-il qu'Archimède ait survécu) dans 'La ruine d'Archimède eut été une perte importante pour la physique attique'. Dans cette phrase, 'La ruine d'Archimède' équivaut grossièrement à 'Le fait qu'Archimède périsse (ait péri)' ['For Archimedes to (have) perish(ed)']. La locution est neutre quant à la réalité de sa ruine. C'est une généralité, un concept, un moyen par lequel nous pouvons représenter-comme. Il ne s'agit pas, comme le suggérait le premier usage, d'une chose dont nous pouvons être témoins. Ce dont il est question ne fait pas partie de l'état des choses ${ }_{\mathrm{m}}$, comme en ferait partie un évènement. (Et ce, même si cette manière de représenter l'état des choses ${ }_{\mathrm{m}}$ fait partie de l'état des choses.) Lorsque ces deux lectures sont confondues, nous attribuons à tort une certaine structure à l'état des choses ${ }_{\mathrm{m}}$, et nous en faisons un objet d'un type qu'il ne peut précisément pas être. 
Il est exact que la pensée selon laquelle Benno est moralisateur se trouve 'dans la réalité elle-même' (si par là on entend simplement que nous pouvons en prédiquer quelque chose). Comme toute pensée, elle n'est pas en tant que telle structurée d'une manière particulière, mais elle est plutôt décomposable en différentes décompositions, qui peuvent chacune la structurer d'une certaine manière. Elle est structurable. Les sous-pensées qui peuvent s'assembler pour former une telle décomposition sont en relation les unes avec les autres, ainsi qu'avec la pensée entière elle-même, via la contribution particulière qu'elles apportent à la manière dont cette pensée entière fait dépendre la vérité de l'état des choses - c'est-à-dire par cet effort partiel qu'elles accomplissent. Cela revient à dire que les sous-pensées se rapportent les unes aux autres (dans le cadre de la structuration d'une pensée) par des relations définies en fonction des demandes qu'elles formulent à l'endroit de la vérité, et des contributions possibles à la satisfaction, au moins partielle, de ces demandes. Benno, lui, se rapporte à ses pairs en vertu de relations définies par sa capacité à les embêter ou à être embêté. Ces deux types de relations ne sont pas les mêmes, et les structures définies par la première ne sont pas les structures définies par la seconde. La vérité ne consiste tout simplement pas en ce second type de correspondance. (Frege, dans (I91 8: 59-60), met fin aux idées autrement) L'argument est valable et supporte l'inclusion de généralités dans la classe des choses qui peuvent être (re)présentées comme tombant elles-mêmes sous des généralités. Je considère que Benoist refuse avec raison la négation de cette idée, à l'endroit précis où les deux grammaires qu'il mentionne seraient confondues.

2. Voici, légèrement reformulée, la deuxième idée avancée par Benoist:

L'essence de la contrainte (logique) que la réalité impose à la pensée tient au fait que dans tout contexte, nous sommes tenus de déterminer la réalité d'une certaine manière. La réalité ne prend une forme déterminée que dans la mesure où nous y déposons certaines manières de la rendre comptable. La seule ontologie est contextualiste.

L'idée est à la fois radicale et profonde. Ainsi conçue, l'ontologie s'intéresse aux formes déterminées, ou aux formes, que prend, ou que peut prendre, la 'réalité' ou ce qui existe (les choses ${ }_{\mathrm{m}}$ ) - quoique, pour faire écho au Wahrsein de Frege, seulement pour autant que la réponse à ces questions nous est donnée par ce que c'est qu'être (quelque chose). Je pense que l'ontologie est même fortement concernée par les deux pôles du représentercomme: par ce qui doit être instancié, comme par ce qui l'instancie. Dans les deux cas, elle s'intéresse à ce qui est réitérable: à une même chose instanciée deux fois, et à une même chose qui instancie deux fois quelque chose; ou aux généralités et à ce qui leur échoit. L'idée de récurrence est intrinsèquement liée à celle d'articulation. Dans chaque cas, il s'agit d'attribuer à quelque chose le rôle d'objet, et je formulerai donc ici la thèse de Benoist en terme d'objectité. 
Commençons avec une idée de Frege. Celui-ci observe que chaque fois qu'on mentionne un objet, l'objet nommé est toujours 'illuminé ('beleuchtet') d'un seul côté à la fois'. Voyons ce qu'implique cette idée de Beleuchtung (d'un certain angle ou d'une certaine perspective). Il est essentiel au fait d'être un objet (ou de jouer le rôle d'un objet) que cet objet ait une carrière/un devenir, ce qui signifie tout simplement qu'il est réitérable, à une fréquence indéfinie. Lorsqu'on mentionne un objet, ce qui identifie cet objet comme étant l'objet en question n'est qu'une étape de sa carrière/ son devenir. L'idée vaut également pour des individus - Pia, Sid et Benno, par exemple - ou pour des choses comme des nombres, des bagues ou des champs. Je ne m'étendrai pas davantage sur ce premier point, sinon pour mentionner qu'il est lié à une notion cruciale chez Frege: le caractère essentiellement public d'une pensée. Quant au second point, Frege l'attribue à notre finitude. Pour un être infini, peut-être, chaque objet serait illuminé de tous les côtés à la fois. Les objets ne seraient pas réitérés, ce qui en éliminerait la nécessité. Pour un tel être, l'articulation des choses en entités dénombrables ne remplirait aucune utilité. La finitude n'est donc pas une simple contingence ici.

Le Beleuchtung nous conduit toutefois à poser une question gênante. Lorsqu'on mentionne un objet, l'objet est beleuchtet d'une seule façon parmi le nombre indéfini de façons dont il pourrait l'être. Considérons donc deux mentions, chacune mentionnant un objet. Supposons que le Beleuchtung soit, dans chaque cas, indépendant de celui de l'autre. Supposons qu'il y ait un objet situé à côté de vous dans le bar, qui chipe des arachides dans votre bol. Celui-là, pourrais-je dire, est embêtant. Imaginons maintenant un objet devant un pupitre, monologuant d'une voix monocorde sur les dernières théories à la mode concernant les tropes. Celui-là, pourrais-je dire, est embêtant. Nul besoin que celui chipant des arachides ait aussi monologué d'une voix monocorde. Nul besoin que celui qui monologuait ait passé la soirée à chiper mes arachides. Dans notre cas, toutefois, avons-nous deux mentions du même objet, deux étapes d'une manière carrière, ou deux mentions différentes d'un objet différent: deux étapes d'une même carrière ou de deux différentes? Nous devons ici nous en tenir aux fondamentaux: la notion d'objet désigne une manière particulière d'articuler les choses $s_{m}$ en entités dénombrables, destinées à servir des fins particulières de la pensée; pour autant qu'il soit question de cette notion, on a affaire à un objet dès lors que celui-ci a un profil cohérent en termes d'identité (dès lors qu'il y a un ensemble cohérent de réponses aux questions demandant si quelque chose $y$ correspond). Ce qui signifie que les choses $\mathrm{m}_{\mathrm{m}}$ peuvent être articulées en un objet pour lequel la réponse à notre question sera 'oui', tout comme elles peuvent être articulées en un objet pour lequel la réponse serait 'non'. Les choses s'articulent en un objet qui a d'abord chipé des arachides, puis a monologué. Mais aussi en un objet qui a d'abord chipé des arachides, a péri, puis a été remplacé par un autre objet qui a monologué. 
Qu'on fixe l'objet et on fixera aussi la réponse aux questions de savoir quand c'est celui-là qui est beleuchet comme ceci ou comme cela. Une fois fixés les évènements en fonction desquels beleuchten, si Sid a chipé, il a aussi monologué. C'est à l'histoire d'en décider. Mais si tout ce que nous savons est qu'un objet est beleuchtet par le fait d'avoir chipé, la notion d'objet ne permet pas à elle seule de décider si l'on peut ou non généraliser à partir de ce cas de façon à y inclure le monologue. Nous pouvons découper un objet dont l'action à un moment précis fut précisément une occurrence de ce vol de cacahouètes (peu importe que le terme 'chiper' soit le mot juste concernant cet objet). Nous pouvons aussi découper l'objet d'une façon (ici) distincte (du point de vue de son objectité). En autorisant la possibilité de ces deux découpages, l'histoire rend eo ipso les deux possibles. Notre exemple peut donc ici servir de base à une induction, dont la conclusion semblerait être la suivante: l'cillumination' qui permet de mentionner un objet autorise du même coup à ce qu'on en mentionne tout un essaim d'autres identiques.

Si nous ne pouvions mentionner que des essaims, la notion d'objectité perdrait sa raison d'être. Nous n'articulons pas sans raison ces essaims en ces entités dénombrables que sont les pensées (Gedanken): nous le faisons en vue d'inférences, qu'elles soient logiques ou reposent sur des connections factives dans le monde. Si nous ne pouvions jamais identifier une pensée, plutôt qu'un simple essaim, nous ne pourrions jamais en mentionner ou en exprimer une, mais seulement un essaim. Ce qui semblerait alors leur enlever tout intérêt. Il semble dès lors que, si le Beleuchtung est intrinsèquement unilatéral, alors il ne suffit pas. (Même si, sans le Beleuchtung, nous ne pourrions pas non plus avoir l'identité.)

Il existe une réponse standard à ce problème. Il est question d'essaims lorsque nous ne possédons pas assez d'informations pour déterminer de quel objet, ou de quel concept, il est question. Il y a un objet qui, à un certain moment, était occupé inter alia à chiper quelque chose. Voilà toute l'information que nous possédons. La question qui se pose maintenant est la suivante: où rencontrons-nous une autre occurrence cet 'cet' objet? La réponse est qu'avec l'information que nous possédons, il est impossible de fournir une réponse unique. Mais supposons maintenant que l'information que nous détenons indique que c'est Sid qui a chipé. Alors, l'indétermination qui pesait sur l'identité de l'objet en question est levée. C'est à ce niveau que Benoist se braque (avec raison). Il ne s'agit pas, nous fait remarquer Benoist, de la bonne manière de disperser un essaim.

De quoi parlons-nous lorsque nous parlons de quelqu'un qui mange du pain avec du miel? Supposons que le 'pain' soit du kougelhopf (un produit à base de levure) et que le miel soit généreusement gorgé de rhum. On pourrait appeler ceci manger du pain avec du miel (si, par exemple, ces items sont exclus du régime de celui qui les mange). Mais on pourrait le refuser (si, par exemple, cette personne a moins de six ans). Ce en quoi consiste le fait, comme tel, de manger du pain avec du miel ne semble donc pas pouvoir, à 
la réflexion, régler ce cas de manière univoque. La question que nous avons posée ne semble donc pas encore être formée de telle sorte qu'elle obtienne une réponse. 'Quand cela a-t-il été dit ?', sommes-nous enclins à demander, 'Dans quelles circonstances?' Selon ce modèle (qu'il faudrait certes davantage justifier), même si nous pouvons dire que les mots (d'une langue donnée) parlent d'une manière dont une chose peut être (dans notre cas: du fait de manger du pain avec du miel), en les utilisant comme nous le faisons pour en parler, nous pouvons aussi parler d'un nombre indéfini d'autres manières dont les choses peuvent être, et la vérité de ce qui est dit sera susceptible de varier en conséquence. On peut comprendre de différentes manières le fait de manger du pain (par exemple).

Considérons maintenant la carrière (légèrement embellie) de Jeremy Bentham, fondateur de University College à Londres et de systèmes juridiques. À sa mort, selon son souhait (et toujours en embellissant l'histoire), il fut embaumé, vêtu d'une tenue de gentleman londonien, placé dans un cercueil de verre et exposé dans l'aula magna de l'université. Notez ici l'usage tout naturel des anaphores 'sa/son/il' dans la dernière phrase. Elles soulèvent une question: est-ce vraiment Bentham qui fut embaumé? Est-ce Bentham qui est désormais exposé? En mentionnant Bentham dans l'expression de notre pensée, nous pouvons être considérés (par des auditeurs appropriés ayant à leur disposition les faits appropriés) comme l'ayant bien mentionné. Mais une chose à laquelle nous pouvons nous résoudre est qu'il n'existe pas de réponse univoque à cette question. De ce fait, en étant qui il est, Bentham (qui est lui-même un concept dont nous ne pouvons pas nous passer) est dans le même bateau, précédemment décrit, que le fait de manger du pain avec du miel.

Voici, donc, une autre manière de disperser les essaims. L'essaim est dispersé dans une certaine mesure lorsqu'il est mentionné que c'est de (ou d'être) Bentham que nous parlons. Il est suffisamment dispersé pour que nous puissions être considérés comme exprimant une seule pensée lorsque nous disons de Bentham qu'il a mangé du pain avec du miel. Mais le nuage n'est dispersé que jusqu'à un certain point, et pas davantage, ce qui signifie que si nous poussons notre questionnement suffisamment loin, nous pouvons toujours atteindre un point où se forme un nouvel essaim (si petit soitil). Ici, la nécessité, mère de l'invention, annonce sa présence. La source du problème réside dans le cœur de l'identité elle-même: la loi de Leibniz. Ce qu'on considère être Bentham peut varier d'une fois à l'autre, mais on ne peut le compter que d'une seule manière à la fois. Nous ne pouvons pas, sans enfreindre cette loi, traiter simultanément Bentham comme un objet qui a bien été embaumé et comme un objet qui pourrait ne pas l'avoir été.

Cette idée ouvre la voie à l'argument de Benoist: ce qui est le cas [what there is], ou, plus exactement, ce que nous pouvons dire de ce qui est le cas, varie avec les occasions qui nous sont données de le dire (si tant est que l'occasion de dire quoi que ce soit se présente). C'est l'idée d'articulation qui 
permet d'exprimer au mieux ce point. Les choses ${ }_{m}$, qui forment elles-mêmes un sujet de prédications (à zéro place), s'articulent d'une grande variété de manières alternatives possibles en d'autres choses (au pluriel) desquelles il pourra être prédiqué quelque chose; mais elles ne s'articulent pas de toutes ces manières à la fois. Les alternatives sont, en règle générale, des alternatives. L'importance de cette idée défendue par Benoist se manifeste également à travers son impact sur la première idée soulevée plus haut. Pourquoi ne pas reconduire les généralités à ce qui les instancie, comme c'était le cas en concevant (plus ou moins métaphoriquement) le caractère moralisateur comme une substance localisée dans des complexes mondains? Parce que (au fond), pour ce qui concerne les manières dont les choses ${ }_{\mathrm{m}}$ peuvent s'instancier, ou les manières dont les choses $\mathrm{m}_{\mathrm{m}}$ (ou les choses) sont en étant ce qu'elles sont, la réalité (les choses $\mathrm{m}_{\mathrm{m}}$ ) demeure rigoureusement neutre. Elle n'impose d'elle-même ni les différentes compréhensions, ni les articulations qu'elle est susceptible d'admettre".

*Traduction par Corinne Lajoie, avec la collaboration de Bruno Ambroise. 